\title{
Quantum Yield of Reflection Mode Varied Doping GaN Photocathode
}

\author{
Jianliang QIAO ${ }^{1, a}$, Xiangjiang $\mathrm{LI}^{1}$, Jun NIU ${ }^{1}$ and Youtang $\mathrm{GAO}^{1}$ \\ ${ }^{1}$ School of Electronic and Electrical Engineering, Nanyang Institute of Technology, NanYang \\ 473004 China \\ aqsyxn@163.com ${ }^{1}$
}

\begin{abstract}
Using the NEA photocathode activation and evaluation experiment system, the varied doping GaN photocathode has been activated and evaluated. According to the diffusion and orientation drifting equation, the quantum yield formula of reflection mode varied doping NEA GaN photocathode was gotten. The factors affecting the quantum efficiency of varied doping GaN photocathode were studied. For the varied doping GaN photocathode, the quantum efficiency is mainly decided by the escape probability of electron $\mathrm{P}$, he absorption coefficient $\alpha$, the electron diffuse length $\mathrm{L}_{\mathrm{D}}$, the reflectance of cathode materials for incident light $\mathrm{R}$, emission layer thickness Te and the inside electric field $E$.The experiment and analysis results show: With the directional inside electric field in the bulk, the varied doping NEA GaN photocathode has better photoemission performance than uniform doping photocathode.
\end{abstract}

\section{Introduction}

With high quantum efficiency, low dark current, concentrated electrons energy distribution etc. characteristics, negative electron affinity (NEA) GaN photocathode has been expected to be an important ultraviolet (UV) photocathode. Princeton University has studied the negative electron affinity characteristic and the electron emission at cesiated $\mathrm{GaN}$ surfaces ${ }^{[1]}$. Northwest University studied the fabrication and low light level applications of GaN photocathode ${ }^{[2,3]}$. University of California studied the development of GaN photocathode for imaging detectors ${ }^{[4,5]}$.Stanford University has done much work in such aspects as preparation of NEA GaN photocathode and surface mechanism in recent years $^{[6,7]}$.

The quantum yield is an important parameter for the preparation and evaluation of NEA $\mathrm{GaN}$ photocathode. How to improve the quantum yield of $\mathrm{GaN}$ photocathode further is still an important study hot focus. With the directional inside electric field in the bulk, the higher quantum efficiency can be obtained for the varied doping GaN photocathode.

* Corresponding author:qsyxn@163.com 


\section{Equation of Quantum Yield for Varied Doping NEA GaN Photocathode}

For the varied doping NEA GaN photocathode, considering the inside electric field $E$ within the material, The diffusion and orientation drifting equation can be expressed as equation $(1)^{[8]}$ :

$$
\frac{d^{2} n_{1}(x)}{d x^{2}}+\frac{\mu|E|}{D_{n}} \frac{d n_{1}(x)}{d x}-\frac{n_{1}(x)}{D_{n} \tau_{1}}+\frac{g_{1}(x)}{D_{n}}=0, x \in\left[0, T_{e}\right]
$$

Where $n_{1}(x)$ is the electron concentration in the emission layer of photocathode, and $\mathrm{x}$ is the distance from some point in the photocathode emission layer to the surface. $\mathrm{D}_{\mathrm{n}}$ is the electron diffuse coefficient, $\mu$ is the electron mobility, $\mathrm{L}_{\mathrm{D}}$ is the electron diffuse length, $\tau_{1}$ is the lifetime of minority carrier in the emission layer, Te is the photocathode emission layer thickness. Where $g_{1}(x)$ is the generation function of photoelectron, and it's expression can be expressed as below:

$$
g_{1}(x)=(1-R) I_{0} \alpha_{1} \exp \left(-\alpha_{1} x\right) .
$$

Where $\mathrm{I}_{0}$ is the intensity of incident light, $\alpha_{1}$ is photocathode absorption coefficient to the incident light, $\mathrm{R}$ is the photocathode reflectivity to the incident light. The border condition is:

$$
\left.n_{1}(x)\right|_{x=0}=0,\left.\quad\left[D_{n} \frac{d n_{1}(x)}{d x}+\mu|E| n_{1}(x)\right]\right|_{x=T_{e}}=-\left.S_{V} n_{1}(x)\right|_{x=T_{e}} .
$$

Where $\mathrm{Sv}$ is the back interface velocity, From solving the above formulas, The expression of quantum efficiency for reflect-mode varied doping NEA GaN photocathode can be gotten ${ }^{[8]}$ :

$$
Y(h v)=\frac{P(1-R) \alpha_{1} L_{D}}{\alpha_{1}^{2} L_{D}{ }^{2}-\alpha_{1} L_{E}-1} \times\left\{\frac{N\left(S-\alpha_{1} D_{n}\right) \exp \left[\left(L_{E} / 2 L_{D}{ }^{2}-\alpha_{1}\right) T_{e}\right]}{M}-\frac{Q}{M}+\alpha_{1} L_{D}\right\} .
$$

Where $L_{E}=\mu|E| \tau=\frac{q|E|}{k_{0} T} L_{D}^{2}, \quad N=\sqrt{L_{E}^{2}+4 L_{D}^{2}}, \quad S=S_{V}+\mu|E|$,

$$
\begin{aligned}
& M=\left(N D_{n} / L_{D}\right) \cosh \left(N T_{e} / 2 L_{D}{ }^{2}\right)+\left(2 S L_{D}-D_{n} L_{E} / L_{D}\right) \sinh \left(N T_{e} / 2 L_{D}{ }^{2}\right), \\
& Q=S N \cosh \left(N T_{e} / 2 L_{D}{ }^{2}\right)+\left(S L_{E}+2 D_{n}\right) \sinh \left(N T_{e} / 2 L_{D}{ }^{2}\right) 。
\end{aligned}
$$

For the uniform doping photocathode, the inside electric field $E$ is zero, then equation (4) can be changed as below ${ }^{[9]}$. 


$$
\begin{aligned}
Y(h v)= & \frac{P(1-R) \alpha_{1} L_{D}}{\alpha_{1}^{2} L_{D}^{2}-1} \times\left[\frac{\left(S_{V}-\alpha D_{n}\right) \exp \left(-\alpha_{1} T_{e}\right)}{\left(D_{n} / L_{D}\right) \cosh \left(T_{e} / L_{D}\right)+S_{V} \sinh \left(T_{e} / L_{D}\right)}\right. \\
& \left.-\frac{S_{V} \cosh \left(T_{e} / L_{D}\right)+\left(D_{n} / L_{D}\right) \sinh \left(T_{e} / L_{D}\right)}{\left(D_{n} / L_{D}\right) \cosh \left(T_{e} / L_{D}\right)+S_{V} \sinh \left(T_{e} / L_{D}\right)}+\alpha_{1} L_{D}\right]
\end{aligned}
$$

If $\mathrm{S}_{\mathrm{V}}=0$ and $\mathrm{T}_{\mathrm{e}}=\infty$, the equation (5) can be simplified:

$$
Y(h v)=\frac{P(1-R)}{1+1 / \alpha_{1} L_{D}} .
$$

\section{Factors Affecting the Quantum Yield}

The main source of NEA photocathode photoemission is the escape of thermalization electron ${ }^{[10]}$. From equation (4),the factors affecting the quantum yield for varied doping NEA GaN photocathode includes the electron diffuse length $L_{D}$, the escape probability of electron $P$, the photocathode absorption coefficient to the incident light $\alpha$, the reflectance of cathode materials for incident light $R$ etc.. In addition, factors affecting the quantum yield for transmission-mode photocathode are also the thickness of photocathode emission layer $T e$ and the back interface velocity $S v$ etc. ${ }^{[11]}$.

(1) escape probability of electron $P$

When the bulk optoelectrons pass across the band bending area and reach to the cathode surface, they can only at a certain probability tunneling surface barrier and escape from the surface, the tunneling probability is the escape probability of electron $P$. $P$ is also known as transmission coefficient tunnelling a certain shape surface barrier for different energy electron. The value of $P$ is related with the energy of the electron transported to cathode surface and the shape of surface barrier after activation. $P$ is one of the important parameters when measuring $\mathrm{GaN}$ photocathode preparation level. The quantum yield theory formula shows that the NEA GaN photocathode quantum yield is increased with the value of $P$.

(2) absorption coefficient $\alpha$ 。

The relationship between the absorption coefficient $\alpha$ of GaN and photon energy is shown in Figure $1^{[12]}$. Within the scope of $\mathrm{GaN}$ threshold $3.4 \mathrm{eV}$ to $5 \mathrm{eV}$, the absorption coefficient increases with decrease of the incident wavelength. For reflection mode NEA $\mathrm{GaN}$ photocathode, we expect $\alpha$ as big as possible. The absorption coefficient $\alpha$ is bigger, the number of the absorption photon near the surface is greater, the photoelectron number close to the surface is more, the quantum yield of cathode is the greater. 


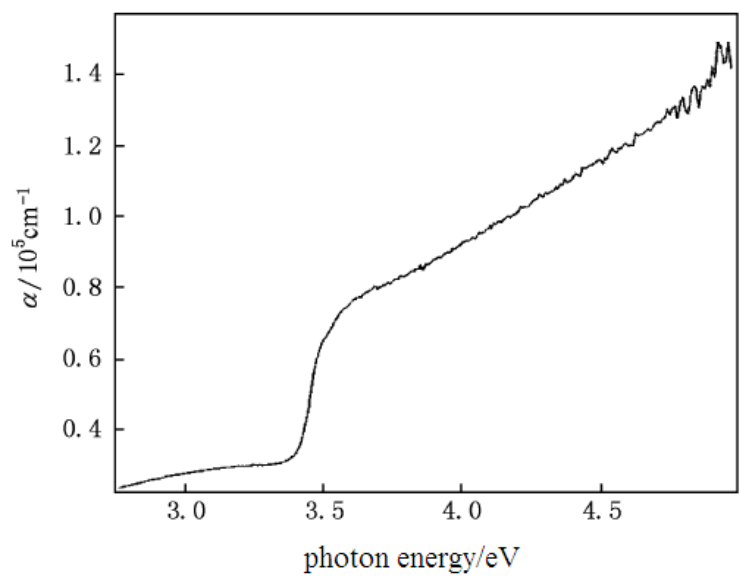

Fig.1 The relationship between the absorption coefficient $\alpha$ of $\mathrm{GaN}$ and photon energy

(3) electron diffuse length $L_{D}$

For NEA GaN photocathode, the greater the electron diffusion length $\mathrm{L}_{\mathrm{D}}$, the more easy the photoelectrons from the bulk in its life period are transported to the cathode surface. And the number of photoelectron to the cathode surface is increase, resulting in higher quantum efficiency.

(4) reflectance of cathode materials for incident light $R$

The photoemission of photocathode belongs to the external photoemission. The direct cause of the photoemission is the incident light. The intensity of the light directly affect the photocurrent, and then affect the final quantum efficiency. So the reflectance of cathode materials for incident light $\mathrm{R}$ is also the important factor influencing the quantum efficiency.

(5) emission layer thickness $T e$

To reflection mode, the cathode is thicker, the photoemission is better, as long as the length of the photon absorption is smaller enough than the electronic absorption length, the photoelectrons can escape.

(6) inside electric field $E$

For the varied doping NEA photocathode, the photoelectrons produced in bulk can be speeded by the inside electric field $E^{[13,14]}$. For the exponential-doping $\mathrm{GaN}$ photocathode, the inside electric field $E$ can be gotten as equation (7) ${ }^{[9]}$ :

$$
E(x)=-\frac{d V(x)}{d x}=-\frac{k_{0} T A}{q}
$$

Where $\mathrm{k}_{0}$ is the boltzmann constant, $\mathrm{T}$ is the absolute temperature, $\mathrm{A}$ is exponential-doping coefficient, and $\mathrm{q}$ is the basic charge constant. The inside electric field $E$ is related to the varied doping NEA photocathode construction.

\section{Evaluation of the Varied Doping NEA GaN Photocathode}

The p-type varied doping GaN sample is doped with $\mathrm{Mg}$, the active layer thickness is about $180 \mathrm{~nm}$. The doping concentration of active layer is respectively about $1.0 \times 10^{18} \mathrm{~cm}^{-3}$, $4.0 \times 10^{17} \mathrm{~cm}^{-3}, 2.0 \times 10^{17} \mathrm{~cm}^{-3}$ and $6.0 \times 10^{16} \mathrm{~cm}^{-3}$ from bulk to surface. The sample was 
activated in the NEA photocathode activation and evaluation experiment system. The quantum efficiency was tested on line after activation. The quantum efficiency curve is shown in Fig. $2^{[15]}$.

At the $\mathrm{GaN}$ threshold $3.4 \mathrm{eV}$, the quantum efficiency curve increase rapidly. In the measurement scope the quantum efficiency reachs the maximum value $56 \%$ at $5.17 \mathrm{eV}$.

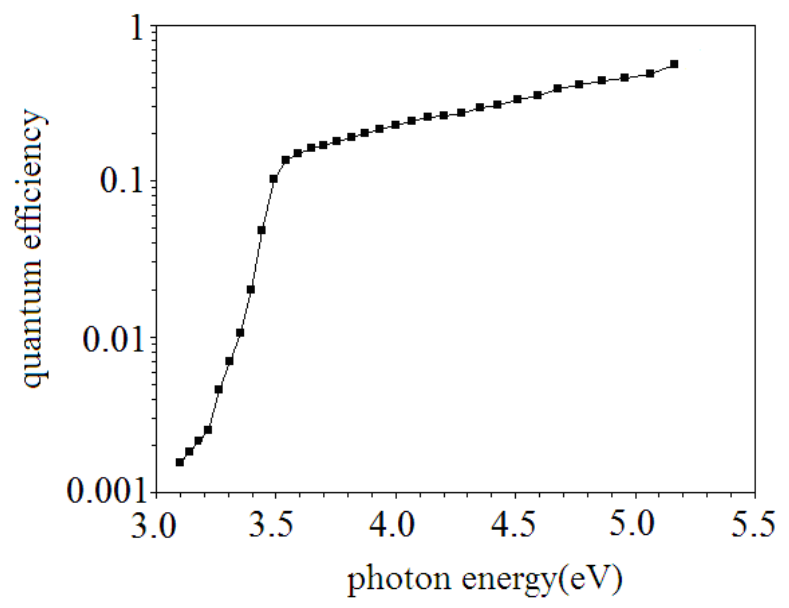

Fig.2 The quantum efficiency curver of the varied doping NEA GaN photocathode

\section{Conclusion}

Because of many virtues, negative electron affinity GaN photocathode is better kind of photocathode. According to the theory of NEA GaN photocathode photoemission, the quantum yield question of the varied doping NEA GaN photocathode was studied. The quantum yield formulas of varied doping NEA GaN photocathode under reflection-mode was given. Using the NEA photocathode activation and evaluation experiment system, the quantum yield curves of the varied doping reflection mode NEA GaN photocathodes were gotten. The results shows: The quantum efficiency for varied doping NEA GaN photocathode at present has already been up to $56 \%$ at $5.17 \mathrm{eV}$ under reflection mode. The influences of performance parameters on the quantum yield were analyzed. The escape probability of electron $P$, he absorption coefficient $\alpha$, the electron diffuse length $\mathrm{L}_{\mathrm{D}}$, the reflectance of cathode materials for incident light $R$, emission layer thickness $T e$ and the inside electric field $E$ are the main factors affecting the quantum yield of the varied doping NEA GaN photocathode.

\section{Acknowledgment}

This work was supported by National Natural Science Foundation of China (Grant No. 61371058). 


\section{References}

1. C.I. Wu, A. Kahn. Negative electron affinity and electron emission at cesiated GaN and AlN surfaces, Applied Surface Science, 162-163,(2000) 250-255.

2. M. P. Ulmer, W. B. Wessels, F. Shahedipour, R. Y. Korotkov, C. Joseph, and T. Nihashi. Progress in the fabrication of GaN photo-cathodes. Proceedings of SPIE,2001, Vol. 4288,246-253.

3. M. P. Ulmer, B. W. Wessels, and O. H. W. Siegmund, Advances in wide-band gap semi-conductor based photocathode devices for low light level applications, Proc. of SPIE, 2002, 4650,94-103.

4. O.H.W. Siegmund, A. S. Tremsin, J. V. Vallerga, J. B. McPhate, J. S. Hull, J. Malloy and A. M. Dabiran, Gallium nitride photocathode development for imaging detectors, Proc. of SPIE, 2008, 7021,70211B.

5. O. Siegmund, J. Vallerga, J. McPhate, J. Malloy, A. Tremsin, A. Martin,M. Ulmer, B. Wessels. Development of GaN photocathodes for UV detectors. Nuclear Instruments and Methods in Physics Research A ,2006,567,89-92.

6. Machuca F, Liu Z, Sun Y, et al. Role of oxygen in semiconductor negative electron affinity photocathodes. J. Vac. Sci. Technol.B. 2003, Vol. 20, No. 6, 2721-2725.

7. Machuca F, Liu Z, Sun Y, et al. Oxygen species in $\mathrm{Cs} / \mathrm{O}$ activated gallium nitride (GaN) negative electron affinity photocathodes. J. Vac. Sci. Technol. 2003, 21(4), 1863-1869.

8. Zou Ji-Jun,Chang Ben-Kang,Yang Zhi. Theoretical calculation of quantum yield for exponential-doping GaAs photocathodes. ACTA PHYSICA SINICA. 2007, 56(5): 2992-2997.

9. Li Biao,Prepration and evaluation of reflection-mode gradient-doping GaN photocathode[D], Nanjing University of Science \& Technology, 2013, 35-40.

10. Jianliang Qiao, Benkang Chang, Yunsheng Qian, Xiaohui Wang, Biao Li, Xiaoqian Fu. "Study on photoemission mechanism for negative electron affinity GaN vacuum electron source", Physica Status Solidi c, 2012,09(01), 36-40.

11. Wang Xiao-Hui, Shi Feng, Guo Hui, Hu Cang-Lu, Cheng Hong-Chang, Chang Ben-Kang, Ren Ling, Du Yu-Jie,Zhang Jun-Ju. "The optimal thickness of a transmission-mode GaN photocathode", Chinese Physics B, 2012,21(8), 087901.

12. Machuca F. A thin film p-type GaN photocathode: prospect for a high performance electron emitter[D], Stanford University, 2003.

13. Zhi Yang, Benkang Chang, and Jijun Zou. Comparison between gradient-doping GaAs photocathode and uniform-doping GaAs photocathode. Applied Optics, 2007, 46(28): 7035-7039.

14. Niu Jun, Zhang Yijun, Chang Benkang, et al. Influence of varied doping structure on photoemissive property of photocathode. Chinese Physics B, 2011, 20(4): 044209.

15. Wang Xiao-Hui, Photoemission performance of wurtzite structure GaN (0001) surface [D], Nanjing University of Science \& Technology, 2013,76. 